SELECCIONES MATEMÁTICAS
Universidad Nacional de Trujillo
ISSN: 2411-1783 (Online)
2019; Vol.06(2): $204-216$.

\title{
Una clase de modelo de depredación del tipo Leslie-Gower con respuesta funcional racional no monotónica y alimento alternativo para los depredadores
}

\section{A class of Leslie-Gower type predator model with a non-monotonic rational functional response and alternative food for the predators}

\author{
Paulo C. Tintinago-Ruiz* (iD) , Lina M. Gallego-BerríotiD , and Eduardo González-Olivares ${ }^{\dagger}$ (iD \\ Received, Jul. 23, 2019 \\ Accepted, Oct. 15, 2019
}

How to cite this article:

Tintinago-Ruiz, P., et al. Una clase de modelo de depredación del tipo Leslie-Gower con respuesta funcional racional no monotónica y alimento alternativo para los depredadores. Selecciones Matemáticas. 2019; 6(2):204-2016. http://dx. doi.org/10.17268/sel .mat.2019.02.07

\section{Resumen}

Las interacciones entre dos especies son básicas en el estudio de cadenas alimentarias complejas, en particular la relación entre los depredadores y sus presas.

El análisis de modelos simples, descritos por sistemas de tiempo continuo, en los cuales se incorporan algunos fenómenos ecológicos dando luces sobre esta interesante interrelación.

En este trabajo, se analiza un modelo de depredador-presa del tipo Leslie-Gower, descrito por un sistema de ecuaciones diferenciales ordinarias (EDO) considerando dos aspectos: la presa se defiende de la depredación, formando grupo de defensa, y los depredadores disponen un alimento alternativo, cuando su alimento favorito escasea. Por lo tanto, se asume una respuesta funcional racional de Holling tipo IV y una modificación de la capacidad de carga de los depredadores para describir estos fenómenos.

Determinamos las condiciones en el espacio de parámetros para la existencia de los equilibrios y la naturaleza de cada uno de ellos.

Concluimos que el parámetro que indica la existencia de alimento alternativo para depredadores tiene una gran importancia en la dinámica del modelo, porque aparecen nuevos puntos de equilibrio y curvas de separatriz en el plano de fase.

Por simulaciones numéricas comprobamos que existe un subconjunto de parámetros para los cuales hay un único punto de equilibrio positivo en el plano de fase, el cual es estable y está rodeado por dos ciclos límites originados por bifurcación de Hopf, el interior inestable y el exterior estable.

Palabras clave. Modelo depredador-presa, respuesta funcional, bifurcación, ciclo límite, curva separatriz, estabilidad.

\footnotetext{
Abstract

The interactions between two species are basic in the study of complex food chains, particularly the relation among the predators and their prey.

The analysis of simple models, described by continuous-time systems, in which some ecological phenomena are incorporated giving lights about this interesting interrelationship.

In this work, a Leslie-Gower type predator-prey model is analyzed, considering two aspects: the prey defends from the predation, forming group defense and the predators have an alternative food. So, a rational Holling type IV functional response and a modification of the predators carrying capacity are assumed, to describe each phenomenon.

We determine conditions on the parameter space for the existence of the equilibria and their nature.
}

\footnotetext{
*Universidad del Quindío, Armenia, Colombia. (t inti27@gmail.com).

†Universidad del Quindío, Armenia, Colombia. ( inag@uniquindio. edu. co).

${ }^{\ddagger}$ Pontificia Universidad Católica de Valparaíso, Chile. (ejgonzal@ucv.cl).
} 
Using the Lyapunov quantities method, we also establish conditions on the parameter values for which there exist a unique positive equilibrium point, which is stable and surrounded by two limit cycle, the innermost unstable and the outermost sable.

We conclude that the parameter indicating the existence of alternative food for predator has a great importance on the dynamic of model, because appear new equilibrum points and separatrix curves in the phase plane.

Some simulations are given to reinforce our findings the ecological interpretations of resultas are given

Keywords. Predator-prey model, functional response, bifurcation, limit cycle, separatrix curve, stability.

1. Introducción. En este trabajo se analiza un modelo de depredador-presa en tiempo continuo, considerando tres aspectos importantes para describir la interacción:

1. La función de crecimiento de los depredadores es de tipo logístico.

2. La respuesta funcional o tasa de consumo de los depredadores es del tipo de Holling IV generalizado.

3. Los depredadores tienen una comida alternativa, cuando escasea su presa favorita.

El primer aspecto caracteriza a los modelos depredador-presa del tipo Leslie o modelo presa depredador logístico [38] o modelos del tipo Leslie-Gower [7], en el cual, la capacidad de carga del medio ambiente del depredador $K_{y}$ es una función del tamaño de presa $x=x(t)$, es decir, depende de los recursos disponibles. En el modelo original propuesto por Philip H. Leslie en 1948 [27], se asume que la capacidad de carga ambiental de los depredadores es proporcional a la abundancia de presas, es decir, $K_{y}=K(x)=n x$, supuesto que también se asume en el modelo de May-Holling-Tanner [6,36] derivado del modelo de Leslie-Gower considerando la respuesta funcional hiperbólica [29].

Este modelos contrastan con los modelos de tipo Gause [13, 25], propuesto por el biólogo ruso Georgii F. Gause en 1934 [16], en los cuales la tasa de conversión de presas consumidas por los depredadores sigue una ley de conservación de masa o energía [9], siendo sistemas de EDO compartimentados [8,38].

En este trabajo, consideramos que la capacidad de carga del medio ambiente del depredador es representada por la función $K_{y}=n x+c[5,22]$, implicando que el depredador es generalista, donde $c>0$ representa la cantidad de alimento alternativo.

El modelo de Leslie-Gower [28] puede generar anomalías en sus predicciones; predice que si la tasa de consumo por depredador per capita es cercana a cero, la población de depredadores puede aumentar, aunque la proporción de presas sea muy pequeña [38]. Sin embargo, este modelo ha sido usado para estudiar algunas interacciones del mundo real [24] y está recibiendo mayor atención de los modeladores $[3,4,30]$.

El objetivo principal de este artículo es estudiar los comportamientos dinámicos del sistema que representa a un modelo de Leslie-Gower considerando .

Por otro lado, uno de los elementos principales de la relación depredador-presa es la respuesta funcional del depredador o la función de consumo. Se refiere al cambio en la densidad de presas atacadas por unidad de tiempo por depredador cuando cambia la densidad de las presas [14,35].

Usualmente las respuestas funcionales dependiente sólo de la población de presas se clasifica en cuatro categorías. Tres de ellas propuestas en un trabajo original de Crawford S. Holling en 1959 [26] y posteriormente R. Taylor [37] incluyó una cuarta categoría. Las más usuales son la lineal $h(x)=q x$, usada en el modelo de Lotka-Volterra [29], la hiperbólica $h(x)=\frac{q x}{x+a}$, empleada en el modelo de May-Holling-Tanner [21,22,36] y la sigmoidea $h(x)=\frac{q x^{2}}{x^{2}+a^{2}}[19,20]$, todas de ellas crecientes y las dos últimas acotadas.

Muchas formas matemáticas se han propuesto para representar la acción de los depredadores sobre las presas y también existen respuestas funcionales dependientes de ambas poblaciones, como la razón-dependiente o la de Bedington-DeAngelis [38].

En este trabajo, utilizamos una respuesta funcional Holling tipo IV generalizada cuya forma es dada por

$$
h(x)=\frac{q x}{x^{2}+b x+a}
$$

con $q>0, a>0$, y $b \in \mathbb{R}$. Como $h(x)>0$, entonces $x^{2}+b x+a>0$; por lo tanto, $b^{2}-4 a<0$, y $-2 \sqrt{a}<b<2 \sqrt{a}$. Además, $h(x)$ tiene un valor maximo cuando $x=\sqrt{a}$

Esta forma de respuesta funcional generaliza la expresada por $h(x)=\frac{q x}{x^{2}+a^{2}}$ que ha sido utilizada en diferentes trabajos anteriores [19,34]. La función $h(x)$ representa una respuesta funcional no monótona que describe un comportamiento antipredador (APB antipredator behavior) denominado formación de grupo de defensa $[1,14,35,41]$.

Grupo de defensa es un término usado para describir el fenómeno por el cual los depredadores disminuyen, o incluso se evitan por completo, debido a la mayor capacidad de la presa para defenderse mejor o cubrir a los más débiles cuando su número es lo suficientemente grande. Para este fenómeno, una respuesta funcional no monotónica describe mejor este comportamiento [2,3,42].

Notamos que $h(x)$ tiende a cero ya que la población de presas tiende a infinito, esto es, $\lim _{x \rightarrow \infty} h(x)=0$. Esto implica que la depredación disminuye cuando el tamaño de la población de presas aumenta. 


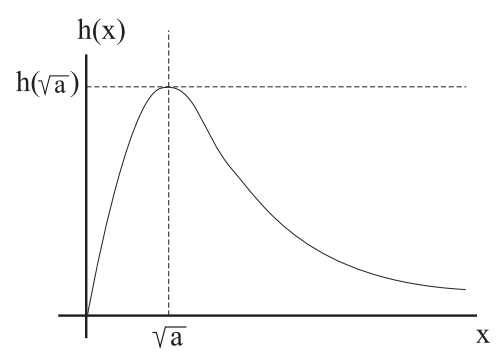

FIGURA 1.1. Respuesta funcional no-monotónica generalizada de Holling tipo IV.

Otra manifestación de un APB descrito por una respuesta funcional no monotónica es el fenómeno de agregación, comportamiento social en el que la presa se congrega en una gran escala en relación con el depredador, de modo que la caza del depredador no es espacialmente homogénea [37], tal como sucede con los grandes cardúmenes de juveniles en ciertas especies de peces.

También hay ejemplos relacionados con el consumo no monótono que se producen a nivel microbiano, donde la evidencia indica que cuando se enfrentan a una sobreabundancia de nutrientes, la efectividad del consumidor puede comenzar a disminuir. Esto se ve a menudo cuando los microorganismos se utilizan para la descomposición de desechos o para la purificación del agua, un fenómeno que es denominado inhibición [14,35,41].

Además, en los últimos años, ha tratado de describir el fenómeno del comportamiento de rebaño de manera similar con el grupo de defensa [39]. Aunque ambos son interacciones sociales entre las presas, no estamos completamente de acuerdo con esta versión de simejanza entre ambos comportamientos [40].

Por otra parte, la existencia, unicidad y la no existencia de ciclos límites son los problemas más importantes relacionados con los modelos bidimensionales para la interacción depredador-presa. El problema de determinar las condiciones, que garantiza la unicidad de un ciclo límite o el número de soluciones periódicas, es una cuestión matemática de importancia.

Conjuntamente con el estudio de la estabilidad global de un único punto de equilibrio positivo único en los modelos depredador-presa, estos problemas han sido ampliamente estudiados en las últimas décadas [25], comenzando con el trabajo de Kuo S. Cheng en 1981 [10]. El fue el primero en demostrar la unicidad de un ciclo límite para un modelo específico de presa depredador considerando una respuesta funcional de tipo II de Holling, utilizando la simetría de la isoclina de las presas.

Este último problema está relacionado con un problema no resuelto propuesto por el matemático David Hilbert en 1900 [15], y se refiere a encontrar el número máximo de ciclos límites de un sistema de ecuaciones diferenciales polinomiales bidimensionales, cuyo grado debe ser igual a $n \in \mathbb{N}$.

2. El modelo. El modelo depredador-presa que se analizará se describe por un sistema de ecuaciones diferenciales de tipo Kolmogorov [8, 13]:

$$
X_{\nu}(x, y):\left\{\begin{aligned}
\frac{d x}{d t} & =\left(r\left(1-\frac{x}{K}\right)-\frac{q y}{x^{2}+b x+a}\right) x \\
\frac{d y}{d t} & =s\left(1-\frac{y}{n x+c}\right) y
\end{aligned}\right.
$$

donde $x(t)$ e $y(t)$ denotan el tamaño de la población de presas y depredadores para $t \geq 0$, respectivamente (medida como número, biomasa o densidades). Todos los parámetros son positivos, es decir, $\nu=(r, K, q, b, a, s, n, c) \in$ $\mathbb{R}_{+}^{8}$, teniendo los siguientes significados biológicos:

- $r$ representa la tasa de crecimiento intrínseca de las presas.,

- $K$ es la capacidad de carga ambiental (o capacidad de soporte) de las presas,

- $q$ es la tasa de ataque per cápita de los depredadores.,

- $a$ y $b$ son parámetros de ajuste de la función $h$,

- $s$ tasa de crecimiento intrínseca de los depredadores,

- $n$ es una medida de la calidad de la presa como alimento, y

- $c$ es el tamaño de alimento alternativo para los depredadores.

El sistema (1) está definido en todo el primer cuadrante, es decir:

$$
\Omega=\left\{(x, y) \in \mathbb{R}^{2} / x \geq 0, y \geq 0\right\}=\mathbb{R}_{0}^{+} \times \mathbb{R}_{0}^{+} .
$$

Los puntos de equilibrio del sistema (1) o singularidades del campo vectorial $X_{\nu}(x, y)$ son: $(0,0),(K, 0)$, $(0, c)$ y $\left(x_{e}, y_{e}\right)$, con $P_{e}$ los puntos de equilibrio positivo, que satisfacen las ecuaciones de las isoclinas $y=n x+c$ e $y=\frac{r}{q}\left(1-\frac{x}{K}\right)\left(x^{2}+b x+a\right)$. 
Entonces, la abscisa $x_{e}$ de los puntos de equilibrio positivos es una solución de la ecuación de tercer grado:

$$
\frac{r}{K q} x^{3}-\frac{r}{q}\left(1-\frac{b}{K}\right) x^{2}+\left(n-\frac{r}{q}\left(b-\frac{a}{K}\right)\right) x-\left(\frac{a r}{q}-c\right)=0 .
$$

Para simplificar los cálculos, seguimos la metodología utilizada en [1, 20,21,36], haciendo un cambio de variables y reescalado el tiempo, lo cual es descrito en la siguiente proposición.

Proposición 1. Sistema topológicamente equivalente

El campo vectorial $X_{\nu}(x, y)$ o sistema (1) es topológicamente equivalente al sistema polinomial del quinto grado.

$$
Y_{\eta}(u, v):\left\{\begin{array}{l}
\frac{d u}{d \tau}=\left((1-u)\left(u^{2}+B u+A\right)-Q v\right)(u+C) u \\
\frac{d v}{d \tau}=S(u+C-v)\left(u^{2}+B u+A\right) v
\end{array}\right.
$$

con $A=\frac{a}{K^{2}}, B=\frac{b}{k}, Q=\frac{q n}{r k^{2}}, S=\frac{s}{r k} y C=\frac{c}{n K}$, donde $\eta=(A, B, Q, S, C) \in \mathbb{R}_{+}^{5}$.

El sistema (2) está también definido en todo el primer cuadrante, es decir:

$$
\bar{\Omega}=\left\{(u, v) \in \mathbb{R}^{2} / u \geq 0, v \geq 0\right\}=\mathbb{R}_{0}^{+} \times \mathbb{R}_{0}^{+} .
$$

Demostración: Sean $x=K u$ e $y=n K v$; reemplazando en el sistema (1) se tiene

$U_{\nu}(u, v):\left\{\begin{aligned} K \frac{d u}{d t} & =\left(r(1-u)-\frac{q n K v}{(K u)^{2}+b K u+a}\right) K u \\ n K \frac{d v}{d t} & =s\left(1-\frac{n K v}{n K u+c}\right) n K v\end{aligned}\right.$

Simplificando, factorizando y simplificando nuevamente, se obtiene

$U_{\nu}(u, v):\left\{\begin{aligned} \frac{d u}{d t} & =r\left((1-u)-\frac{q n v}{r K\left(u^{2}+\frac{b}{K} u+\frac{a}{K^{2}}\right)}\right) u \\ \frac{d v}{d t} & =s\left(1-\frac{v}{\left(u+\frac{c}{n K}\right)}\right) v\end{aligned}\right.$

Considerando el reescalamiento del tiempo dado por

$$
t=\frac{1}{r}\left(u+\frac{c}{n K}\right)\left(u^{2}+\frac{b}{K} u+\frac{a}{K^{2}}\right) \tau,
$$

se obtiene

$\frac{d u}{d t}=\frac{d u}{d \tau} \frac{d \tau}{d t}=\frac{d u}{d \tau} \frac{r}{\left(u+\frac{c}{n K}\right)\left(u^{2}+\frac{b}{K} u+\frac{a}{K^{2}}\right)} \mathrm{y}$ análogamente $\frac{d v}{d t}=\frac{d v}{d \tau} \frac{r}{\left(u+\frac{c}{n K}\right)\left(u^{2}+\frac{b}{K} u+\frac{a}{K^{2}}\right)}$.

Reemplazando se obtiene

$V_{\nu}(u, v):\left\{\begin{array}{l}\frac{d u}{d \tau} \frac{r}{\left(u+\frac{c}{n k K}\right)\left(u^{2}+\frac{b}{K} u+\frac{a}{K^{2}}\right)}=r\left((1-u)-\frac{q n v}{r K\left(u^{2}+\frac{b}{K} u+\frac{a}{K^{2}}\right)}\right) u \\ \frac{d v}{d \tau} \frac{r\left(1-\frac{v}{\left(u+\frac{c}{n K}\right)}\right) v}{\left(u+\frac{c}{n K}\right)\left(u^{2}+\frac{b}{K} u+\frac{a}{K^{2}}\right)}=s\left(1-\frac{c}{2}\right)\end{array}\right.$

y finalmente

$V_{\nu}(u, v):\left\{\begin{array}{l}\frac{d u}{d \tau}=\left((1-u)\left(u^{2}+\frac{b}{K} u+\frac{a}{K^{2}}\right)-\frac{q n v}{r K}\right)\left(u+\frac{c}{n K}\right) u \\ \frac{d v}{d \tau}=\frac{s}{r}\left(u+\frac{c}{n K}-v\right)\left(u^{2}+\frac{b}{K} u+\frac{a}{K^{2}}\right) v\end{array}\right.$

Definiendo $A=\frac{a}{K^{2}}, Q=\frac{q n}{r K}, B=\frac{b}{K}, S=\frac{s}{r}$ y $C=\frac{c}{n K}$ se obtiene el sistema (2).

Nota 1. Con el cambio efectuado se ha construído un difeomorfismo $\Psi: \bar{\Omega} \times \mathbb{R} \rightarrow \Omega \times \mathbb{R}$, tal que $\Psi(u, v, \tau)=\left(K u, n K v, \frac{1}{r}\left(u+\frac{c}{n K}\right)\left(u^{2}+\frac{b}{K} u+\frac{a}{K^{2}}\right) \tau\right)=(x, y, t)$

La matriz Jacobiana de $\Psi$ es

$$
D \Psi(u, v, \tau)=\left(\begin{array}{ccc}
K & 0 & 0 \\
0 & n K & 0 \\
D \Psi(u, v, \tau)_{31} & 0 & \frac{1}{r}\left(u+\frac{c}{n K}\right)\left(u^{2}+\frac{b}{K} u+\frac{a}{K^{2}}\right)
\end{array}\right)
$$

$D \Psi(u, v, \tau)_{31}=\frac{1}{K^{2} n r}\left(b c+a n+3 K^{2} n u^{2}+2 K c u+2 K b n u\right) \tau$

luego,

$\operatorname{det} D \Psi(u, v, \tau)=\frac{n K^{2}}{r}\left(u+\frac{c}{n K}\right)\left(u^{2}+\frac{b}{K} u+\frac{a}{K^{2}}\right)>0$.

Entonces, $\Psi$ es un difeomorfismo que preserva la orientación del tiempo [11, 12], para el cual el campo vectorial (1) en el nuevo sistema de coordenadas, es topológicamente equivalente al campo vectorial $Y_{\eta}=\Psi \circ$ $X_{\nu}$; toma la forma $Y_{\eta}=P(u, v) \frac{\partial}{\partial u}+Q(u, v) \frac{\partial}{\partial v}[12]$ y el sistema de ecuaciones diferenciales asociado está dado por el sistema (2) que es polinomial de quinto orden.

Los puntos de equilibrio del sistema (2) o singularidades del campo vectorial $Y_{\eta}(u, v)$ son $(0,0),(1,0)$, $(0, C)$ los puntos de equilibrio positivo $\left(u_{e}, v_{e}\right)$, que satisfacen las ecuaciones de las isoclinas $v=u+C \mathrm{y}$ $v=\frac{1}{Q}(1-u)\left(u^{2}+B u+A\right)$. 
Luego, $v_{e}=u_{e}+C$ donde $u_{e}$ es la abscisa de los puntos de equilibrio positivos; luego, es una solución de la ecuación de tercer grado:

$$
P(u)=u^{3}-(1-B) u^{2}+(A-B+Q) u-(A-C Q)=0 .
$$

Basados en la regla de signos de Descartes, para el polinomio $P(u)$ se pueden presentar 27 casos diferentes de posibles soluciones de acuerdo a los signos de los coeficientes de la ecuación (3). Puede tener una raíz positiva real única o tres raíces positivas reales diferentes o dos raíces positivas reales distintas, una de ellas con multiplicidad dos

Asumiendo que $A-C Q \neq 0$, sea $u_{e}=H$ la raíz real positiva que siempre existe para la ecuación (3). Asumiendo que $H<1$, y efectuando la división entre $P(u)$ y $(u-H)$ obtenemos el polinomio cuadrático

$$
P_{1}(u)=u^{2}-(1-B-H) u+A-B+Q-(1-B-H) H
$$

como factor de $P(u)$ y el resto de la división es

$$
R(H, A, B, Q)=H^{3}-(1-B) H^{2}+(A-B+Q) H-(A-C Q)=0 .
$$

De esta última relación podemos despejar uno de los parámetros en función de $H$ y los restantes parámetros. Por ejemplo $Q=\frac{-H^{3}+(1-B) H^{2}+(B-A) H+A}{C+H}$.

Como $Q>0$, entonces $-H^{3}+(1-B) H^{2}+(B-A) H+A>0$, esto es, $A>\frac{H^{3}-(1-B) H^{2}-B H}{1-H}$.

Si $H<1$, entonces $H^{3}-(1-B) H^{2}-B H>0$.

Reemplazando $Q$ en $P_{1}(u)$ obtenemos

$$
P_{1}(u)=u^{2}-(1-B-H) u+\frac{A(1+C)-C(1-H)(B+H)}{C+H} .
$$

Definamos

$$
\begin{aligned}
& a_{1}=1-B-H, a_{0}=\frac{A(1+C)-C(1-H)(B+H)}{C+H} \mathrm{y} \\
& \Delta=a_{1}^{2}-4 a_{0}=\frac{H^{3}+(2 B-3 C-2) H^{2}+\left(2 C-2 B-2 B C+B^{2}+1\right) H+\left(C B^{2}+2 C B-4 A+C-4 A C\right)}{C+H} .
\end{aligned}
$$

Lema 1. Para la ecuación (4), se tiene que:

1. Asumiendo $a_{1}>0$ y $a_{0}>0$; entonces

1.1 existen dos soluciones reales positivas, si y sólo si, $\Delta>0$, las cuales son

tales que $u_{1}<u_{2}$.

$$
u_{1}=\frac{1}{2}\left(a_{1}-\sqrt{\Delta}\right) y u_{2}=\frac{1}{2}\left(a_{1}+\sqrt{\Delta}\right),
$$

1.2 existe una solución real positiva de multiplicidad 2 , si y sólo si, $\Delta=0$.

Esta solución es $u^{*}=\frac{1}{2}(1-B-H)$.

1.3 No tiene raíces reales positivas, si y sólo si, $\Delta<0$.

2. No existen raíces reales positivas, si y sólo si,

$$
\begin{aligned}
& 2.1 a_{1}=0 \text { y } a_{0}>0, o \\
& 2.2 a_{1}<0 \text { y } a_{0}>0 .
\end{aligned}
$$

3 Existe una solución real positiva, si y sólo si, $a_{1}<0$ y $a_{0}<0$.

Demostración: Es inmediato $\square$

Supongamos que $A-C Q=0$. La ecuación (3) se reduce a

$$
P_{0}(u)=\left(u^{2}-(1-B) u+(A-B+Q)\right) u=0 .
$$

Sean $b_{1}=1-B$ y $b_{2}=A-B+Q$ y $\Delta_{0}=(1-B)^{2}-4(A-B+Q)$.

Notamos que cuando $u=0$, uno de los puntos de equilibrio positivo coincide con el punto $(0, C)$.

Lema 2. Para la ecuación (5), se tiene:

1. Asumiendo $b_{1}=1-B>0$ y $b_{0}=A-B+Q>0$; entonces existe

1.1 dos soluciones reales positivas, si y sólo si, $\Delta_{0}>0$.

Son $u_{10}=\frac{1}{2}\left(b_{1}-\sqrt{\Delta_{0}}\right)$ y $u_{20}=\frac{1}{2}\left(b_{1}+\sqrt{\Delta_{0}}\right)$, tales que $u_{10}<u_{20}$.

1.2 una solución real positiva de multiplicidad 2 , si y sólo si, $\Delta_{0}=0$.

Esta solución es $u^{* *}=\frac{1}{2}(1-B)$. 
1.3 No tiene raíces reales positivas, si y sólo si, $\Delta_{0}<0$.

2. No existen raíces reales positivas, si y sólo si,

$2.1 b_{1}=0$ y $b_{0}>0, o$

$2.2 b_{1}<0$ y $b_{0}>0$.

Demostración: También es inmediato. dos:

3. Resultados Principales. Para el sistema (2) o campo vectorial $Y_{\eta}(u, v)$, tenemos los siguientes resulta-

Lema 3. Existencia de una region de invarianza

El conjunto $\bar{\Lambda}=\left\{(u, v) \in \mathbb{R}^{2} / 0 \leq u \leq 1, v \geq 0\right\} \subset \bar{\Omega}$ es una region positivamente invariante.

Demostración: Como el sistema (2) es de tipo Kolmogorov, entonces los ejes coordenados son conjuntos invariantes [11].

Sea $u=1$; tenemos que $\frac{d u}{d \tau}=-Q v(1+C)<0$.

Luego, para cualquiera sea el signo de la segunda ecuación

$$
\frac{d v}{d \tau}=S(1+C-v)(1+B+A) v .
$$

Se tiene que las trayectorias o soluciones entran a la región $\bar{\Gamma}$ y las que tienen condiciones iniciales en $\bar{\Gamma}$, permanece allí. te.

Para el sistema (1) el conjunto $\Lambda=\left\{(x, y) \in \mathbb{R}^{2}: 0 \leq x \leq K, y \geq 0\right\}$ es una region positivamente invarian-

Además, para el sistema (2) existe una subregión de invarianza determinada por la isoclina de los depredadores,

$$
\overline{\Lambda_{1}}=\left\{(u, v) \in \mathbb{R}^{2}: 0 \leq u \leq 1, v \leq u+C\right\} .
$$

Lema 4. Acotamiento de las soluciones

Las soluciones son acotadas

Demostración: Usando la compactificación de Poincaré [32].

Sean $X=\frac{u}{v}$ e $Y=\frac{1}{v}$; luego, $\frac{d X}{d \tau}=\frac{1}{v^{2}}\left(v \frac{d u}{d \tau}-u \frac{d v}{d \tau}\right)$ y $\frac{d Y}{d \tau}=-\frac{1}{v^{2}} \frac{d v}{d \tau}$.

Entonces el sistema (2) toma la forma:

$$
\tilde{Y}_{\eta}(X, Y)\left\{\begin{array}{ccc} 
& \\
\frac{d X}{d \tau}= & \frac{1}{Y^{4}}\left(\begin{array}{c}
X^{4} Y-X^{5}-B X^{4} Y-C X^{4} Y+S X^{3} Y-S X^{4} Y \\
+A X^{2} Y^{3}-A X^{3} Y^{2}+B X^{3} Y^{2}+C X^{3} Y^{2}-Q X^{2} Y^{2} \\
+A C X Y^{4}+A S X Y^{3}-C Q X Y^{3}-A C X^{2} Y^{3}+B C X^{2} Y^{3} \\
-B C X^{3} Y^{2}-A S X^{2} Y^{3}+B S X^{2} Y^{2}-B S X^{3} Y^{2} \\
-C S X^{3} Y^{2}-A C S+X Y^{4}-B C S X^{2} Y^{3} \\
\frac{d Y}{d \tau}=
\end{array}\right. \\
\quad-S(X+C Y-1) \frac{A Y^{2}+X^{2}+B X Y}{Y^{2}}
\end{array}\right.
$$

Es fácil ver que la matriz Jacobiana del campo vectorial $\tilde{Y}_{\eta}(X, Y)$ no está definida en $(0,0)$. Mediante el reescalamiento $T=\frac{1}{Y^{4}} \tau$ obtenemos

$$
\breve{Y}_{\eta}(X, Y)\left\{\begin{array}{c}
X^{4} Y-X^{5}-B X^{4} Y-C X^{4} Y+S X^{3} Y-S X^{4} Y \\
+A X^{2} Y^{3}-A X^{3} Y^{2}+B X^{3} Y^{2}+C X^{3} Y^{2}-Q X^{2} Y^{2} \\
\frac{d X}{d T}=\quad+A C X Y^{4}+A S Y^{3}-C Q X Y^{3}-A C X^{2} Y^{3}+B C X^{2} Y^{3} \\
\\
\\
\\
\\
\end{array}\right.
$$

La matriz Jacobiana del campo vectorial $\breve{Y}_{\eta}(X, Y)$ es

$$
D \breve{Y}_{\eta}(0,0)=\left(\begin{array}{ll}
0 & 0 \\
0 & 0
\end{array}\right) \text {. }
$$

Para desingularizar el origen, empleamos el método del blowing-up direccional [12], mediante el cambio de variables $X=r$ and $Y=r^{2} s$. Así obtenemos

$$
V_{\eta}(r, s):\left\{\begin{array}{l}
\frac{d r}{d T}=\frac{d r}{d T} \\
\frac{d s}{d T}=\frac{1}{r^{2}}\left(\frac{d Y}{d T}-2 r s \frac{d r}{d T}\right)
\end{array}\right.
$$


Reemplazando en $\breve{Y}_{\eta}(X, Y)$ se obtiene,

$$
V_{\eta}(r, s):\left\{\begin{array}{c}
\frac{d r}{d T}=r^{5}\left(\begin{array}{c}
S s+r s-Q r s^{2}-A r^{2} s^{2}+A r^{3} s^{3}+B r^{2} s^{2}+C r^{2} s^{2} \\
-B r s-C r s-S r s+B S r s^{2}-A C r^{3} s^{3}-B C r^{2} s^{2}+A C r^{4} s^{4} \\
+B C r^{3} s^{3}+A S r^{2} s^{3}-C Q r^{2} s^{3}-A S r^{3} s^{3}-B S r^{2} s^{2}-C S r^{2} s^{2} \\
-A C S r^{4} s^{4}-B C S r^{3} s^{3}-1
\end{array}\right) \\
\frac{d Y}{d T}=r^{4} s\left(\begin{array}{c}
-S s-2 r s+2 Q r s^{2}+2 A r^{2} s^{2}-2 A r^{3} s^{3}-2 B r^{2} s^{2} \\
-2 C r^{2} s^{2}+2 B r s+2 C r s+S r s-B S r s^{2}+2 A C r^{3} s^{3} \\
+2 B C r^{2} s^{2}-2 A C r^{4} s^{4}-2 B C r^{3} s^{3}-A S r^{2} s^{3}+2 C Q r^{2} s^{3}+A S r^{3} s^{3} \\
+B S r^{2} s^{2}+C S r^{2} s^{2}+A C S r^{4} s^{4}+B C S r^{3} s^{3}+2
\end{array}\right)
\end{array}\right.
$$

Haciendo un nuevo reescalamiento de la variable independiente (el tiempo) dado por $\lambda=r^{4} T$, se obtiene el nuevo campo vectorial

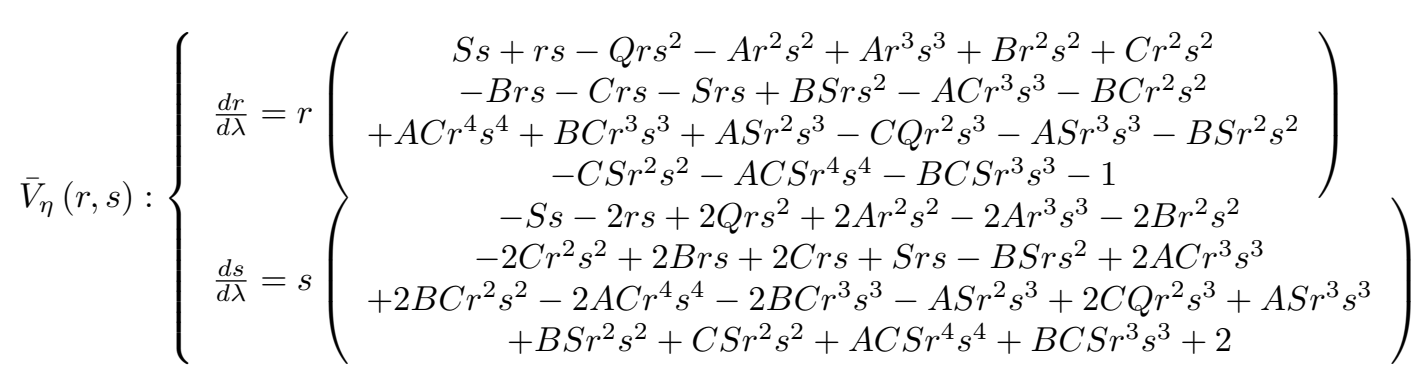

Evaluando la matriz Jacobiana en el punto $(0,0)$ se obtiene

$$
D \bar{V}_{\eta}(0,0)=\left(\begin{array}{cc}
-1 & 0 \\
0 & 2
\end{array}\right) .
$$

Por lo tanto, $(0,0)$ es un punto silla hiperbólico del campo vectorial $\bar{V}_{\eta}(r, s)$; luego, $(0,0)$ es un punto silla no-hiperbólico de los campos vectoriales $V_{\eta}(r, s)$ e $\tilde{Y}_{\eta}(X, Y)$; entonces, el punto $(0, \infty)$ es un punto silla en el campo vectorial compactificado $Y_{\eta}(u, v)$, siendo repulsor por el eje vertical.

Luego, las soluciones son acotadas. $\square$

Nota 2. El resultado anterior nos indica que el sistema (1) está bien propuesto, desde el punto de vista ecológico, porque no pueden haber una gran cantidad de depredadores y una cantidad reducida de presas, para que subsista la interacción.

Para los siguientes resultados requerimos la matriz Jacobiana del sistema (2), que es dada por:

$$
\begin{aligned}
& \qquad D Y_{\eta}(u, v)=\left(\begin{array}{cc}
D Y_{\eta}(u, v)_{11} & -Q u(C+u) \\
D Y_{\eta}(u, v)_{21} & S(C+u-2 v)\left(u^{2}+B u+A\right)
\end{array}\right) \\
& \text { con } \\
& D Y_{\eta}(u, v)_{11}=D Y_{\eta}(u, v)_{11 a}+D Y_{\eta}(u, v)_{11 b}+\left(B-A+2 u-2 B u-3 u^{2}\right)(u+C) u, \\
& \text { donde } \\
& \qquad D Y_{\eta}(u, v)_{11 a}=\left((1-u)\left(u^{2}+B u+A\right)-Q v\right)(u+C), \mathrm{y} \\
& \qquad D Y_{\eta}(u, v)_{11 b}=\left((1-u)\left(u^{2}+B u+A\right)-Q v\right) u
\end{aligned}
$$

(derivada de un triple producto). Además.

$D Y_{\eta}(u, v)_{21}=S v\left(A+B C+2 B u-B v+2 C u-2 u v+3 u^{2}\right)$

3.1. Naturaleza de los puntos de equilibrio sobre los ejes. A continuación será determinada la estabilidad de cada punto de equilibrio en los ejes coordenados.

Lema 5. Naturaleza de $(0,0)$

El punto de equilibrio $(0,0)$ es un repulsor para todo valor de parámetros.

Demostración: Evaluando la matriz Jacobiana en $(0,0)$, se tiene

$$
D Y_{\eta}(0,0)=\left(\begin{array}{cc}
A C & 0 \\
0 & A S C
\end{array}\right) .
$$

Luego, det $D Y_{\eta}(0, C)=A^{2} C^{2} S>0$ y como la traza de la matriz es $\operatorname{tr} D Y_{\eta}(0,0)=S(A C)^{2}>0$, se tiene que el punto $(0,0)$ es repulsor. $\square$

Lema 6. Naturaleza de $(1,0)$

El punto de equilibrio $(1,0)$ es una silla hiperbólica para todo valor de parámetros. 
Demostración: Evaluando la matriz Jacobiana en $(1,0)$, se tiene

$$
D Y_{\eta}(1,0)=\left(\begin{array}{cc}
-(C+1)(A+B+1) & -Q(C+1) \\
0 & S(C+1)(A+B+1)
\end{array}\right) .
$$

Claramente, $\operatorname{det} D Y_{\eta}(1,0)=-S(C+1)^{2}(A+B+1)^{2}<0$; luego, el punto $(1,0)$ es una silla hiperbólica.

Lema 7. Naturaleza de $(0, C)$

El punto de equilibrio $(0, C)$ es

i) una silla hiperbólica, si y sólo si, $A-C Q>0$,

ii) una silla no-hiperbólica, si y sólo si, $A-C Q=0$,

iii) un atractor hiperbólico, si y sólo si, $A-C Q<0$.

Demostración: Evaluando la matriz Jacobiana en $(0, C)$ se tiene

$$
D Y_{\eta}(0, C)=\left(\begin{array}{cc}
C(A-C Q) & 0 \\
S A C & -S A C
\end{array}\right) .
$$

Claramente, si

i) $A-C Q>0$, si y sólo si, $\operatorname{det} D Y_{\eta}(0, C)=-S A C^{2}(A-C Q)<0$, y $(0, C)$ es una silla hiperbólica.

ii) $A-C Q=0$, si y sólo si, $\operatorname{det} D Y_{\eta}(0, C)=0$, y $(0, C)$ es una silla no-hiperbólica (usando el Teorema de la Variedad Central [23]).

iii) $A-C Q<0$, si y sólo si, $\operatorname{det} D Y_{\eta}(0, C)=S A C^{2}(A-C Q)>0$; por lo tanto, la naturaleza de $(0, C)$ depende de la traza y se tiene que $\operatorname{tr} D Y_{\eta}(0, C)=C(A-C Q)-S A C<0$. $\square$

Nota 3. El término $A-C Q$ en la ecuación (3) expresa la relación entre la ordenada del punto de equilibrio $(0, C)$ y el intercepto $\frac{A}{Q}$ de la isoclina de las presas con el eje vertical (eje $\left.-v\right)$.

Entonces, el punto de equilibrio $(0, C)$ es

i) una silla hiperbólica, si y sólo si, $\frac{A}{Q}>C$,

ii) una silla no-hiperbólica, si y sólo si, $\frac{A}{Q}=0$,

iii) un atractor hiperbólico, si y sólo si, $\frac{A}{Q}<C$.

3.2. Existencia de curva heteroclínica. En lo que sigue asumiremos que $A-C Q>0$, esto es, $(0, C)$ es una silla hiperbólica, y que existe un único punto de equilibrio al interior de la región de invarianza $\bar{\Lambda}$, que denotaremos $(H, H+C)$. mente.

Denotaremos por $W^{s}(0, C)$ y $W^{u}(0, C)$ las variedades estable e inestable del punto silla $(0, C)$, respectiva-

Nota 4. La variedad estable $W_{+}^{s}(0, C)$ del punto $(0, C)$ origina una curva $\bar{\Sigma}$, cuyo $\alpha$-limite puede estar dentro o fuera de la región $\bar{\Lambda}$, divide el comportamiento de las trayectorias en el plano de fase. Aquellas con condiciones iniciales por sobre la curva $\bar{\Sigma}$ tienen al punto $(0, C)$ como su $\omega$-limite; aquellas trayectorias con condiciones iniciales por debajo de $\bar{\Sigma}$ pueden tener diferentes $\omega$-limites.

La curva separatriz $\bar{\Sigma}$, la línea recta $u=1$ y el eje u determina una subregión $\bar{\Gamma}$, que es cerrada y acotada,

$$
\tilde{\Gamma}=\left\{(u, v) \in \mathbb{R}^{2}: 0 \leq u \leq 1,0 \leq v \leq v_{\bar{\Sigma}} \text { tal que }\left(u, v_{\bar{\Sigma}}\right) \in \bar{\Sigma}\right\}
$$

i.e., una región compacta donde aplica el Teorema de Poincaré Bendixson.

Teorema 1. Existencia de curva heteroclínica

Sean $W_{+}^{s}(0, C)$ y $W^{u}(1,0)$ la variedades estable e inestable de los puntos sillas $(0, C)$ y $(1,0)$, repectivamente. Entonces, existe un subconjunto de parámetros para las cuales las variedades intersectan, esto es, $W_{+}^{s}(0, C) \cap W_{+}^{u}(1,0) \neq \phi$, dando origen a un ciclo heteroclinico $\gamma_{0}$ uniendo los puntos $(0, C) y(1,0)$.

Demostración: Es claro que la curva determinada por la variedad inestable $W^{u}(1,0)$ permanece en $\bar{\Lambda}$ y es acotada; su $\omega$-limite es el único punto de equilibrio positivo o un ciclo límite rodeando ese punto o el punto $(0, C)$.

Sean $\left(u^{*}, v_{s}\right) \in W^{s}(0, C)$ y $\left(u^{*}, v_{u}\right) \in W^{u}(1,0)$ dos puntos en el plano de fase, con $0<u^{*}<1$, donde $v_{s}$ y $v_{u}$ son funciones de los parámetros $A, B, Q$ y $S$, i.e., $v_{s}=f_{1}(A, B, Q, S)$ y $v_{u}=f_{2}(A, B, Q, S)$.

Es claro que si $0<u^{*}<<1$, entonces $v_{s}<v_{u}$; si $0<<u^{*}<1$, entonces $v_{s}>v_{u}$.

Como el campo vectorial $Y_{\eta}(u, v)$ es continuo respecto a los valores de los parámetros, entonces las variedades estable e inestable se intersectan; por lo tanto, existen $\left(u^{*}, v^{* s}\right)$ y $\left(u^{*}, v^{* u}\right) \in \bar{\Lambda}$ tal que $v^{* s}=v^{* u}$ y la ecuación $f_{1}(A, B, Q, S)=f_{2}(A, B, Q, S)$ define una superficie en el espacio de parámetros para la cual la curva heteroclínica $\gamma$ existe y el grafo $\gamma_{0}$ uniendo los puntos $(0, C)$ y $(1,0)$. $\square$ 


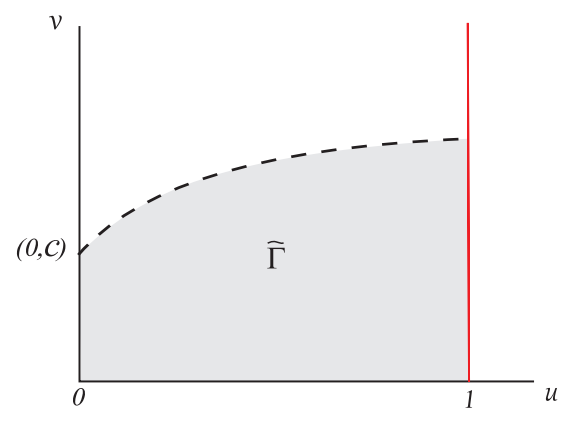

FIGURA 3.1. Región compacta donde es posible aplicar el Teorema de Poincaré-Bendixon.
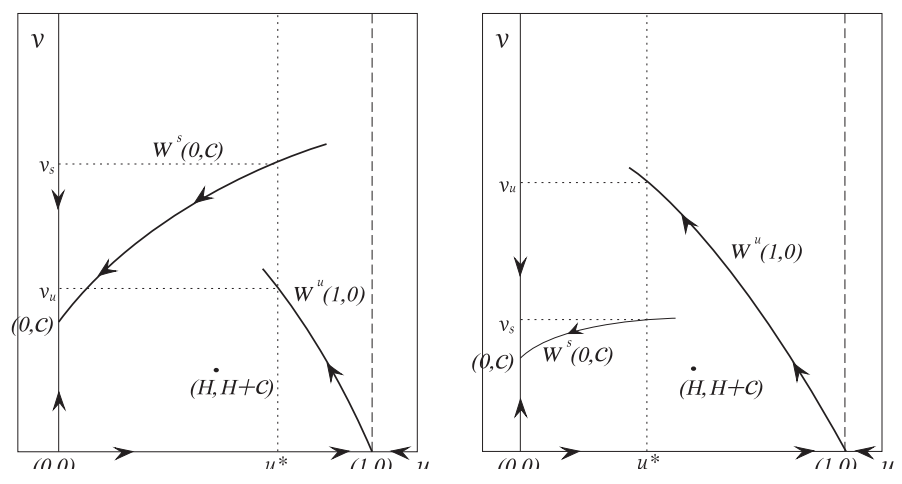

FIGURA 3.2. Posiciones relativas de la variedad estable $W_{+}^{s}(0, C)$ y de la variedad inestable $W^{u}(1,0)$.

En la siguiente figura se muestra las posiciones relativas de las variedades estable $W_{+}^{s}(0, C)$ e inestable $W^{u}(1,0)$.

3.3. Naturaleza del punto de equilibrio positivo. La naturaleza del punto de equilibrio $(H, H+C)$ depende de la relación entre $v_{u}$ y $v_{s}$, las ordenadas de los puntos $\left(u, v_{s}\right) \in W^{s}(0, C)$ y $\left(u, v_{u}\right) \in W^{u}(1,0)$, respectivamente y además de la matriz Jacobiana evaluada en el punto $(H, H+C)$, que es dada por:

$$
\begin{aligned}
& D Y_{\eta}(H, H+C)=\left(\begin{array}{cc}
\left(B-A+2 H-2 B H-3 H^{2}\right) H(C+H) & -Q H(C+H) \\
S(C+H)\left(H^{2}+B H+A\right) & -S(C+H)\left(H^{2}+B H+A\right)
\end{array}\right) . \\
& \text { Luego, } \\
& \quad \operatorname{det} D Y_{\eta}(H, H+C)=H S(C+H)^{2}\left(A+B H+H^{2}\right)\left(A-B-2 H+Q+2 B H+3 H^{2}\right) \\
& \quad \operatorname{tr} D Y_{\eta}(H, H+C)=(C+H)\left(\left(B-A+2 H-2 B H-3 H^{2}\right) H-S\left(H^{2}+B H+A\right)\right)
\end{aligned}
$$

Como ya se ha demostrado que $(0,0)$ es repulsor, $(1,0)$ es silla y $(0, C)$ es también silla. Asumiendo que $(H, H+C)$ está en la región compacta $\bar{\Lambda}$, entonces asumiremos que $A-B-2 H+Q+2 B H+3 H^{2}>0$; por lo tanto, $\operatorname{det} D Y_{\eta}(H, H+C)>0$ y la naturaleza de depende del signo de la traza, es decir, depende del factor

$$
T=\left(B-A+2 H-2 B H-3 H^{2}\right) H-S\left(H^{2}+B H+A\right)
$$

Denotaremos por $P=\left(\operatorname{tr} D Y_{\theta}(H, H+C)\right)^{2}-4 \operatorname{det} D Y_{\theta}(H, H+C)$ para determinar si el equilibrio positivo es foco o nodo.

Teorema 2. Naturaleza del punto de equilibrio positivo

1. Supongamos que $v_{s}>v_{u}$, entonces,

1.1 Es un atractor hiperbólico, si y sólo si, $S>\frac{\left(B-A+2 H-2 B H-3 H^{2}\right) H}{H^{2}+B H+A}$. Mas aún,

1.la es un nodo atractor, si y sólo si, $P>0$.

$1.1 b$ es un foco atractor, si y sólo si, $P<0$.

1.2 Es un repulsor hiperbólico, si y sólo si, $S<\frac{\left(B-A+2 H-2 B H-3 H^{2}\right) H}{H^{2}+B H+A}$. Mas aún,

1.2 a es un nodo repulsor, si y sólo si, $P>0$.

$1.2 b$ es un foco repulsor, si y sólo si, $P<0$. Además, existe al menos un ciclo límite rodeando al punto.

1.3 Es un foco débil (equilibrio no-hiperbólico), si y sólo si, $S=\frac{\left(B-A+2 H-2 B H-3 H^{2}\right) H}{H^{2}+B H+A}$. 
2. Supongamos que $v_{s}<v_{u}$, entonces, $(H, H+C)$ es un nodo o foco repulsor hiperbólico y las trayectorias del sistema (2) tienen al punto $(0, C)$ como su $\omega$-limite, siendo un equilibrio casi globalmente estable [31,33].

Demostración: La demostración de 1 es inmediata usando la matriz Jacobiana.

En el caso 2, existe un ciclo límite, cuyo diámetro aumenta hasta coincidir con la curva heteroclínica que une los puntos de equilibrio $(0, C)$ y $(1,0)$.

Posteriormente, al cambiar los parámetros en forma continua, esta heteroclínica se rompe y la singularidad $(0, C)$ es un atractor para casi todas las trayectorias, salvo el punto $(H, H+C)$.

Corolario 1. Existe una bifurcación de Hopf cuando $S=\frac{\left(B-A+2 H-2 B H-3 H^{2}\right) H}{H^{2}+B H+A}$.

Demostración: Cuando el punto $(H, H+C)$ es un foco repulsor, por Teorema de Poincaré Bendixson, debe existir un ciclo límite rodeando al punto de equilibrio. Además, se cumple la condición de transversalidad [11], pues.

$$
\frac{\partial}{\partial S}\left(\operatorname{tr} D Y_{\theta}(H, H+C)\right)=-\left(H^{2}+B H+A\right)<0 . \square
$$

Nota 5. La debilidad del foco cuando $S=\frac{\left(B-A+2 H-2 B H-3 H^{2}\right) H}{H^{2}+B H+A}$, debe ser determinada. Una manera es calculando las primeras cantidades (o constantes) de Lyapunov [11], pero eso no se presentará en el presente trabajo. Sin embargo, simulaciones numéricas muestran que al menos existen dos ciclos límites concéntricos (infinitesimales), rodeando el punto $(H, H+C)$, existiendo fenómeno de bi-estabilidad.

4. Simulaciones numéricas. Para reforzar nuestros resultados analíticos mostramos ejemplos usando el paquete computacional Pplane.

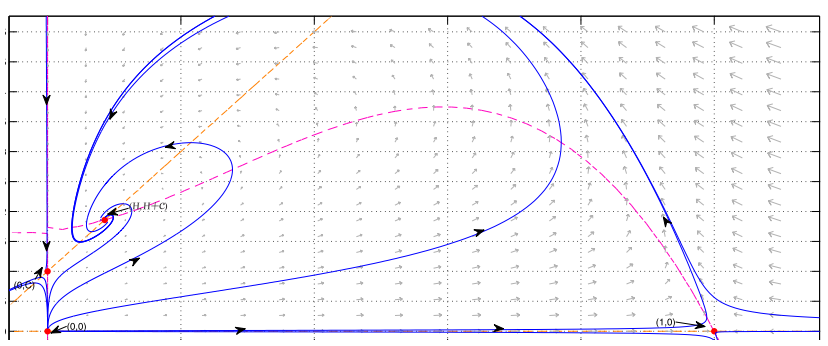

FIGURA 4.1. El único punto de equilibrio positivo es un nodo atractior.

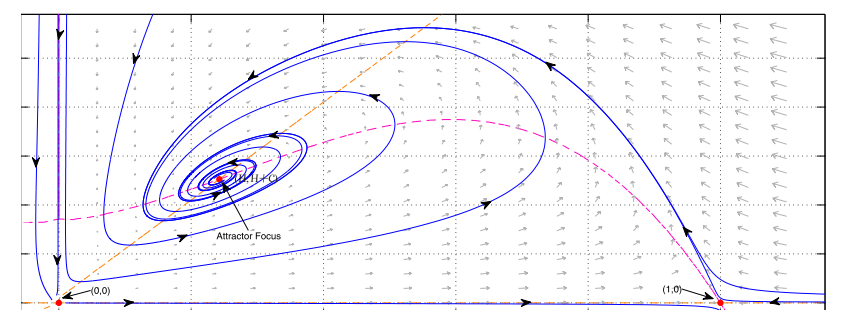

FIGURA 4.2. El único punto de equilibrio positivo es un foco atractor 


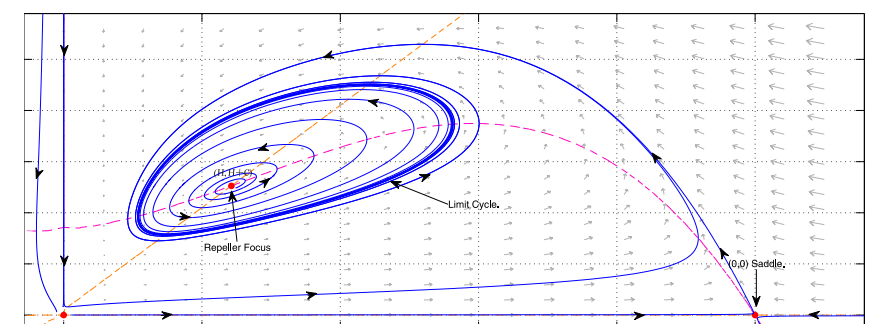

FIGURA 4.3. El único punto de equilibrio positivo es un foco repulsor rodeado de un ciclo límite estable.

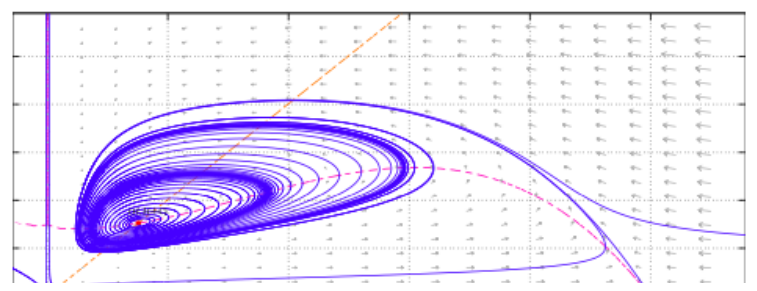

FIGURA 4.4. El único punto de equilibrio positivo es un foco atractor, rodeado por dos ciclos límite, el interior inestable y el exterior estable. Existe el fenómeno de bi-estabilidad.

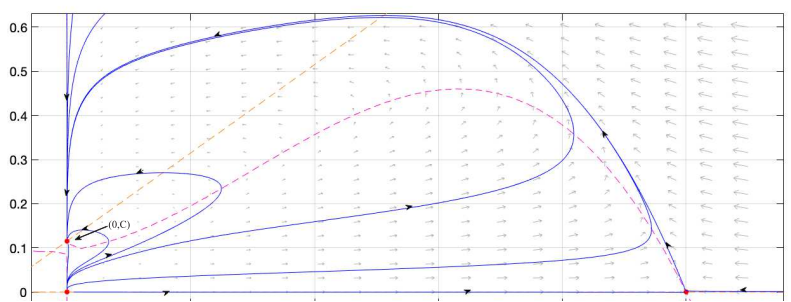

FIGURA 4.5. El único punto de equilibrio esta muy cerca de $(0, C)$, siendo este punto casi globalmente estable [31, 33].

5. Conclusions. En este trabajo hemos mostrado las principales propiedades de un modelo de depredación del tipo Leslie-Gower, asumiendo que los depredadores disponen de un alimento alternativo, en caso que su presa favorita sea escasa. También hemos asumido que las presas ejercen un comportamiento antipredatorio llamado formación de grupo de defensa.

Mediante un difeomorfismo, obtuvimos unl sistema topológicamente equivalente al original, dependiente sólo de cuatro parámetros. Mostramos que en este modelo (y en el original) hay situaciones dinámicas diferentes según la relación entre los parámetros $A, C$ y $Q$, específicamente en el término $A-Q C$. Sin embargo, analizamos sólo el caso en que existe un único punto de equilibrio positivo.

Entre los resultados más importantes, se ha demostrado la existencia de una curva separatriz $\bar{\Sigma}$, dividiendo el comportamiento de las soluciones o trayectorias del sistema. Esto implica que para un mismo conjunto de parámetros dos soluciones muy cercanas pero a diferente lado de esa separatriz $\bar{\Sigma}$, tendrán diferentes y alejados $\omega$-límites, lo cual significa que las soluciones son altamente sensibles a las condiciones iniciales.

Cuando la relación presa/depredador es pequeña (muchos depredadores y pocas presas) implicaría la extinción de la población de presas y el tamaño de la población de los depredadores tendería a alcanzar su máxima capacidad de carga medioambiental, pues el punto $(0, C)$ es atractor para todas las trayectorias con condición inicial por encima de la curva $\bar{\Sigma}$.

Sin embargo, existe una región $\bar{\Lambda}$ en el espacio de fase donde las especies coexisten en el largo tiempo. Si las condiciones iniciales de los tamaños de población están por debajo de $\bar{\Sigma}$, las especies no pueden ir a la extinción 
simultáneamente, pues el punto de equilibrio $(0,0)$ es un repulsor para todo valor de parámetros.

Así mismo, el punto $(1,0)$ es silla hiperbólica, lo cual implica que la interacción se mantiene aún cuando exista una poca cantidad de depredadores. Además, en la región $\bar{\Lambda}$, los tamaños poblacionales pueden oscilar alrededor de un punto de tamaños fijos $(H, H+C)$ por un largo tiempo o coexistir para esos tamaños de población (cuando el punto es atractor).

Entonces en el sistema estudiado se presenta el fenómeno de bi-estabilidad, pues para una misma condición de parámetros, las trayectorias pueden converger al punto $(0, C)$ y al mismo tiempo otras convergen a un punto de equilibrio positivo atractor $(H, H+C)$ o bien, a un ciclo límite estable.

Las oscilaciones se mantendrán hasta que el ciclo límite creado por bifurcación de Hopf aumente su diámetro hasta coincidir con la curva heteroclínica que une los puntos sillas $(0,0)$ y $(1,0)$. Cuando se rompe la heteroclínica (existiendo una bifurcación heteroclínica), el punto $(0, C)$ pasa a ser un atractor casi global [31,33], siendo $(H, H+C)$ la única solución que no converge a $(0, C)$.

Otras situaciones dinámicas del sistema (1), serán presentadas a futuro en una extensión del presente trabajo.

En resumen, el modelo de depredación del tipo Leslie-Gower modificado, considerando un depredador generalista y una función de consumo no-monótonica, tiene gran riqueza matemática y un comportamiento ecológico interesante para los modeladores.

6. Acknowledgements. PTR y LMGB fueron parcialmente financiados por Proyecto interno de la Universidad del Quindío, Armenia, Colombia.

\section{ORCIID and license}

Paulo C. Tintinago-Ruiz https:// orcid.org/0000-0002-4100-2155,

Lina Gallego https: / / orcid.org/0000-0002-5758-9421,

Eduardo Gonzáles-Olivares https://orcid.org/0000-0003-3907-0076.

This work is licensed under the Creative Commons Attribution-NoComercial-ShareAlike 4.0.

Referencias

[1] Aguilera-Moya A. and González-Olivares E. A Gause type model with a generalized class of non-monotonic functional response, In R. Mondaini (ed.), Proceedings of the Third Brazilian Symposium on Mathematical and Computational Biology, E-Papers Serviços Editoriais, Ltda., Rio de Janeiro, 2004; 2:206-217.

[2] Aguilera-Moya A., González-Yañez B. and González-Olivares E. Existence of multiple limit cycles on a predator-prey with generalized non-monotonic functional response, In R. Mondaini (ed.), Proceedings of the Fourth Brazilian Symposium on Mathematical and Computational Biology, E-Papers Serviços Editoriais, Ltda., Rio de Janeiro, 2005; 2:196-210.

[3] Aguirre, P., González-Olivares, E., Sáez, E. Two limit cycles in a Leslie-Gower predator-prey model with additive Allee effect, Nonlinear Analysis: Real World Applications, 2009; 10:1401-1416.

[4] Aguirre,P., González-Olivares, E., Sáez, E. Three limit cycles in a Leslie-Gower predator-prey model with additive Allee effect, SIAM Journal on Applied Mathematics, 2009; 69(5):1244-1269.

[5] Arancibia-Ibarra, C. and González-Olivares, E. A modified Leslie-Gower predator-prey model with hyperbolic functional response and Allee effect on prey, In R. Mondaini (Ed.) BIOMAT 2010. International Symposium on Mathematical and Computational Biology, World Scientific Co. Pte. Ltd., Singapore (2011) 146-162.

[6] Arrowsmith, D. K. and Place,C. M. Dynamical System.Differential equations, maps and chaotic behaviour, Chapman and Hall, 1992.

[7] Aziz-Alaoui, M. A. and Daher Okiye, M. Boundedness and global stability for a predator-prey model with modied Leslie-Gower and Holling-type II schemes, Applied Mathematics Letters, 2003; 16: 1069-1075.

[8] Bazykin, A.D. Nonlinear Dynamics of interacting populations, World Scientific Publishing Co. Pte. Ltd., 1998.

[9] A. A. Berryman, A.A., Gutierrez, A.P. and Arditi, R. Credible, parsimonious and useful predator-prey models - A reply to Abrams, Gleeson, and Sarnelle, Ecology, 1995; 76:1980-1985.

[10] Cheng, K. S. Uniqueness of a limit cycle for a predator-prey system, SIAM Journal of Mathematical Analysis. 1981; 12:541-548.

[11] Chicone, C. Ordinary differential equations with applications, Texts in Applied Mathematics, 34, Springer, 1999.

[12] Dumortier, F., Llibre, J. and Artés, J. C. Qualitative theory of planar differential systems, Springer, 2006.

[13] H. I. Freedman, Deterministic mathematical models in populations ecology, Marcel Dekker, Inc. New York 1980.

[14] Freedman, H. I. and Wolkowicz, G. S. K. Predator-prey systems with group defence: The paradox of enrichment revisted. Bulletin of Mathematical Biology. 1986; 48:493-508.

[15] Gaiko, V. A. Global Bifurcation theory and Hilbert's sixteenth problem, Mathematics and its Applications 559, Kluwer Academic Publishers, 2003.

[16] Gause, G. F. The Struggle for existence, Dover, 1934.

[17] González-Olivares, E. A predator-prey model with nonmonotonic consumption function, In R. Mondaini (Ed.) Proceedings of the Second Brazilian Symposium on Mathematical and Computational Biology, E-papers Serviços Editoriais Ltda. Río de Janeiro, (2003) 2339.

[18] González-Olivares, E., Mena-Lorca, J., Rojas-Palma, A. and Flores, J. D. Dynamical complexities in the Leslie-Gower predator-prey model considering a simple form to the Allee effect on prey, Applied Mathematical Modelling, 2011; 35:366-381.

[19] González-Olivares, E. and Rojas-Palma, A. Allee effect in Gause type predator-prey models: Existence of multiple attractors, limit cycles and separatrix curves. A brief review, Mathematical Modelling of Natural Phenomena. 2013; 8(6):143-164.

[20] González-Olivares, E., Tintinago-Ruiz, P. and Rojas-Palma, A. A Leslie-Gower type predator-prey model with sigmoid funcional response, International Journal of Computer Mathematics, 2015; 93(9):1895-1909.

[21] González-Olivares, E., Gallego-Berrío, L. M., González-Yañez, B. and Rojas-Palma,A. Consequences of weak Allee effect on prey in the May-Holling-Tanner predator-prey model, Mathematical Methods in the Applied Sciences, 2016; 39:4700-4712. 
[22] González-Olivares, E., Arancibia-Ibarra, C., Rojas-Palma, A. and González-Yañez, B. Bifurcations and multistability on the MayHolling-Tanner predation model considering alternative food for the predators, Mathematical Biosciences and Engineering, 2019; 16(5):4274-4298.

[23] Guckenheimer J. and Holmes, P. Nonlinear Oscillations, Dynamical Systems, and Bifurcations of Vector Fields, Springer-Verlag, 1983.

[24] Hanski, I., Hentonnen, H., Korpimaki, E., Oksanen, L. and Turchin, P. Small-rodent dynamics and predation, Ecology, 2001; 82:15051520 .

[25] Hasík, K. On a predator-prey system of Gause type, Journal of Mathematical Biology, 1010; 60:59-74.

[26] Holling, C. S. The components of predation as revealed by a study of small-mammal predation of the European pine sawfly, The Canadian Entomologist, 1959; XCI:293-320.

[27] Leslie, P. H. Some further notes on the use of matrices in population mathematics, Biometrika, 1948; 35:213-245.

[28] Leslie, P. H., Gower, J. C. The properties of a stochastic model for the predator-prey type of interaction between two species, Biometrika, $1960 ; 47: 219-234$

[29] May, R. M. Stability and complexity in model ecosystems, Princeton University Press, 1974.

[30] Martínez-Jeraldo, N. and Aguirre, P. Allee effect acting on the prey species in a Leslie-Gower predation model, Nonlinear Analysis: Real World Applications, 2019; 45:895-917.

[31] Monzón, P. Almost global attraction in planar systems, System and Control Letter, 2005; 54:753-758.

[32] Perko, L. Differential equations and dynamical systems, Springer-Verlag, 1991.

[33] Rantzer, A. A dual to Lyapunov's stability theorem, System and Control Letter, 2001; 42:161-168.

[34] Rojas-Palma A. and González-Olivares, E. Gause type predator-prey models with a generalized rational non-monotonic functional response, In J. Vigo-Aguiar (Ed.) Proceedings of the 14th International Conference on Computational and Mathematical Methods in Science and Engineering, CMMSE 2014, 4:1092-1103. ISBN: 978-84-616-9216-3.

[35] Ruan, S. and Xiao, D. Global analysis in a predator-prey system with nonmonotonic functional response, SIAM Journal on Applied. Mathematics, 2001; 61:1445-1472.

[36] Sáez, E. González-Olivares, E. Dynamics on a Predator-prey Model, SIAM Journal of Applied Mathematics, 1999; 59(5):1867-1878.

[37] Taylor, R. J. Predation, Chapman and Hall, 1984.

[38] Turchin, P. Mongraphs in population biology: Vol. 35. Complex population dynamics. A theoretical/empirical synthesis, 2003.

[39] Venturino, E. and Petrovskii, S. Spatiotemporal behavior of a prey-predator system with a group defense for prey, Ecological Complexity, $2013 ; 14: 37-47$

[40] Vilches, K., González-Olivares, E. and Rojas-Palma, A. Prey herd behavior modeled by a generic non-differentiable functional response, Mathematical Modelling of Natural Phenomena, 2018; 13:26.

[41] Wolkowicz, G. S. W. Bifurcation analysis of a predator-prey system involving group defense, SIAM Journal on Applied Mathematics, 1988; 48:592-606.

[42] Zhu, H., Campbell, S. A. and Wolkowicz, G. S. K. Bifurcation analysis of a predator-prey system with nonmonotonic functional response, SIAM Journal on Applied Mathematics, 2002; 63:636-682. 